\title{
PERDA ELETROLÍTICA DE CÁLCIO, MAGNÉSIO E FERRO NO SUOR DURANTE CORRIDA EM ESTEIRA
}

\author{
ELECTROLYTIC LOSS OF CALCIUM, MAGNESIUM AND IRON IN THE SWEAT DURING RUNNING \\ INA TREADMILL
}

Artigo Original

Original Article

Artículo Original

\author{
PÉRDIDA ELECTROLITICA DE CALCIO, MAGNESIO Y HIERRO EN EL SUDOR DURANTE CARRERA \\ EN CINTA RODANTE
}

\section{Fabrícia Geralda Ferreira' (Profissional de Educação Física) Marina Fassarella² (Nutricionista) Neuza Maria Brunoro Costa ${ }^{3}$ (Nutricionista) \\ Ângela Maria Campos Santana² (Nutricionista) \\ João Carlos Bouzas Marins ${ }^{2}$ (Profissional de Educação Física) \\ 1. Escola Preparatória de Cadetes do Ar (EPCAR), Barbacena, MG, Brasil. \\ 2. Universidade Federal de Viçosa (UFV), Viçosa, MG, Brasil. \\ 3. Universidade Federal do Espírito Santo (UFES), Alegre, ES, Brasil.}

\section{Correspondência:}

Universidade Federal de Viçosa, Departamento de Educação Física, Laboratório de Performance Humana. Av. P.H. Rolfs, s/n, Campus Universitário, Viçosa, MG, Brasil. 36570-000. fafege@yahoo.com.br

\section{RESUMO}

Introdução: O suor e sua consequente evaporação são fundamentais para manutenção da temperatura corporal durante o exercício. Objetivo: Avaliar a perda de cálcio $\left(\mathrm{Ca}^{++}\right)$, magnésio $\left(\mathrm{Mg}^{++}\right)$e ferro $\left(\mathrm{Fe}^{++}\right)$no suor de corredores e de indivíduos ativos. Métodos: Foram avaliados 15 atletas corredores de fundo $\left\{\mathrm{VO}_{2 \text { máx }}=\right.$ $\left.68 \pm 5,4 \mathrm{ml}(\mathrm{kg} \cdot \mathrm{min})^{-1}\right\}$ e 15 indivíduos ativos não atletas $\left\{\mathrm{VO}_{2 \operatorname{máx}}=50,3 \pm 6,3 \mathrm{ml}(\mathrm{kg} \cdot \mathrm{min})^{-1}\right\}$, com média de idade, respectivamente, de 25,3 $\pm 2,4$ e 23,1 \pm 4,3 anos. Ambos os grupos se exercitaram por 80 minutos em esteira, com intensidade de $75 \%$ a $85 \%$ da frequência cardíaca de reserva, e ingeriram $3 \mathrm{ml}$ de água $/ \mathrm{kg}$ de peso corporal a cada 15 minutos. As condições ambientais da prova foram $21,9 \pm 1,5^{\circ} \mathrm{C}$ e 89,2 $\pm 5,6 \%$ de umidade relativa para os atletas e $21,8 \pm 1,6^{\circ} \mathrm{C}$ e $93,2 \pm 3,5 \%$ de UR para os ativos. As amostras de suor foram coletadas em intervalos regulares de 20 minutos nas regiões do peito, torácica e lombar das costas, para posterior análise dos minerais $\mathrm{Ca}^{++}, \mathrm{Mg}^{++}$e Fe ${ }^{++}$por espectrofotômetro de absorção atômica. Resultados: Não foram registradas diferenças significativas para os minerais em função do nível de condicionamento. Observou-se tendência à diminuição na concentração do $\mathrm{Mg}^{++}$e Fe${ }^{++}$do suor ao longo do exercício. Conclusão: Nas condições ambientais e de exercício estudadas, o condicionamento não interfere na perda de $\mathrm{Ca}^{++}, \mathrm{Mg}^{++}$e Fe ${ }^{++}$.

Descritores: hidratação; minerais; exercício.

\section{ABSTRACT}

Introduction: Sweat and its consequent evaporation are essential for maintaining body temperature during the exercise. Objective: To evaluate the loss of calcium $\left(\mathrm{Ca}^{++}\right)$, magnesium $\left(\mathrm{Mg}^{++}\right)$and iron $\left(\mathrm{Fe}^{++}\right)$in the sweat of runners and active individuals. Methods: Fifteen long-distance-runners $\left\{\mathrm{VO}_{2 \max }=68 \pm 5.4 \mathrm{ml}(\mathrm{kg} \cdot \mathrm{min})^{-1}\right\}$ and 15 non-athletes active subjects $\left\{V_{2 m a x}=50.3 \pm 6.3 \mathrm{ml}(\mathrm{kg} \mathrm{min})^{-1}\right\}$ with mean ages of $25.3 \pm 2.4$ and $23.1 \pm 4.3$ years, respectively. Both groups exercised for 80 minutes on a treadmill, with intensity of $75-85 \%$ of reserve heart rate, and ingested $3 \mathrm{ml}$ of water $/ \mathrm{kg}$ of body weight every 15 minutes. The environmental conditions of the test were $21.9 \pm 1.5^{\circ} \mathrm{C}$ and $89.2 \pm 5.6 \%$ relative humidity for athletes and $21.8 \pm 1.6{ }^{\circ} \mathrm{C}$ and $93.2 \pm 3.5 \% \mathrm{RH}$ for active individuals. The sweat samples were collected at regular intervals of 20 minutes in the chest, thoracic and lower back regions for further analysis of the minerals $\mathrm{Ca}^{++}, \mathrm{Mg}^{++}$and $\mathrm{Fe}++$ by atomic absorption spectrophotometer. Results: No significant differences were recorded for the minerals in relation to fitness level. There was a tendency for a decrease in $\mathrm{Mg}^{++}$and $\mathrm{Fe}^{++}$concentrations of sweat over the course of the exercise. Conclusion: In the environmental and exercise conditions studied, the conditioning does not interfere in the loss of $\mathrm{Ca}^{++}, \mathrm{Mg}^{++}$and $\mathrm{Fe}^{++}$.

Keywords: fluid therapy; minerals; exercise.

\section{RESUMEN}

Introducción: El sudor y su consecuente evaporación son esenciales para mantener la temperatura corporal durante el ejercicio. Objetivo: Evaluar la pérdida de calcio $\left(\mathrm{Ca}^{++}\right)$, magnesio $\left(\mathrm{Mg}^{++}\right)$y hierro $\left(\mathrm{Fe}^{++}\right)$en el sudor en corredores y personas activas. Métodos: Se evaluaron 15 corredores de larga distancia $\left\{\mathrm{VO}_{2 \text { máx }}=68 \pm 5,4 \mathrm{ml}\left(\mathrm{kg} \cdot \mathrm{min}^{-1}\right\}\right.$ y 15 individuos activos no atletas $\left\{V_{2} O_{\text {máx }}=50,3 \pm 6,3 \mathrm{ml}(\mathrm{kg} \cdot \mathrm{min})^{-1}\right\}$, con promedio deedades de, respectivamente, $25,3 \pm 2,4$ e 23,1 \pm 4,3 años. Ambos grupos hicieron ejercicio durante 80 minutos en una cinta rodante con una intensidad del $75 \%$ al $85 \%$ de la frecuencia cardiaca de reserva, siendo hidratados con $3 \mathrm{ml}$ de agua/ $/ \mathrm{Kg}$ de peso corporal cada 15 minutos. Las condiciones ambientales de la prueba fueron $21,9 \pm 1,5^{\circ} \mathrm{C}$ y $89,2 \pm 5,6 \%$ de humedad relativa para los atletas y $21,8 \pm 1,6^{\circ} \mathrm{C}$ y $93,2 \pm 3,5 \%$ de HR para los sujetos activos. Las muestras de sudor se recogieron a intervalos regulares de 20 minutos en las regiones del pecho, torácica y lumbar de la espalda para el análisis del $\mathrm{Ca}^{++}, \mathrm{Mg}^{++}$y Fe ${ }^{++}$por espectrofotometría de absorción atómica. Resultados: No se observaron diferencias significativas para los minerales en función del nivel de acondicionamiento. Hubo una tendencia a la disminución de la concentración de $\mathrm{Mg}^{++}$y Fe ${ }^{++}$en el sudor durante el ejercicio. Conclusión: En el ejercicio estudiado y las condiciones ambientales, el acondicionamiento no interfiere en la pérdida de $\mathrm{Ca}^{++}, \mathrm{Mg}^{++}$y Fe $e^{++}$.

Descriptores: fluidoterapia; minerales; ejercicio. 


\section{INTRODUÇÃO}

O suor e sua consequente evaporação são fundamentais para manutenção da temperatura corporal durante o exercício. Sua composição apresenta diferentes elementos, entre eles os minerais cálcio $\left(\mathrm{Ca}^{++}\right)$, magnésio $\left(\mathrm{Mg}^{++}\right)$e ferro $\left(\mathrm{Fe}^{++}\right)^{1}$. Embora a necessidade do organismo para estes minerais seja pequena, quantidades adequadas devem ser fornecidas para a manutenção das funções fisiológicas².

É comum encontrar baixo consumo de alguns minerais, principalmente o $\mathrm{Ca}^{++}$e o $\mathrm{Fe}^{++}$entre atletas ${ }^{3-7}$, assim, caso haja entre os indivíduos uma perda elevada, a ocorrência de deficiências poderá ser facilitada. Desta forma, investigar a perda destes minerais por meio do suor torna-se importante.

Apesar de haver uma quantidade considerável de estudos que examinaram a secreção dos minerais no suor, a maior parte deles avaliaram os minerais sódio $\left(\mathrm{Na}^{+}\right)$e potássio $\left(\mathrm{K}^{+}\right)^{8-10}$ inviabilizando a definição do comportamento dos elementos traços no exercício.

Neste contexto, estudos que buscam avaliar a perda no suor dos elementos traços são necessários, a fim de verificar os fatores que interferem na sua excreção e assim, estabelecer estratégias nutricionais mais adequadas. Entre os possíveis fatores de interferência temos o nível de condicionamento, o tempo de exercício e a diferença na perda mineral entre as regiões corporais, não sendo possível atribuir a perda total do corpo ao que ocorre em uma região específica ${ }^{11-12}$.

Desta forma, os objetivos do presente estudo foram: a) avaliar se condicionamento físico interfere na perda dos minerais $\mathrm{Ca}^{++}, \mathrm{Mg}^{++} \mathrm{eFe}^{++} \mathrm{em}$ três regiões corporais-peito, costas região torácica e costas região lombardurante 80 minutos de corrida intermitente e b) avaliar se a composição do suor, nas três regiões, sofre modificações no seu efeito tempo e região corporal, de acordo com o nível de condicionamento físico dos avaliados.

\section{MÉTODOS}

O estudo foi aprovado pelo Comitê de Ética em Pesquisa com Seres Humanos da Universidade Federal de Viçosa (UFV), MG, Brasil (parecer $\left.n^{\circ} 035 / 2006\right)$. Foram avaliados 15 atletas corredores de fundo e 15 indivíduos ativos não atletas, sendo este número determinado baseando-se em estudos de mesma natureza, como o estudo desenvolvido por Amano et al. ${ }^{13}$ Os voluntários após receberem esclarecimentos assinaram o Termo de Consentimento Livre e Esclarecido.

Para todos voluntários foram considerados como critério de inclusão: serem do sexo masculino com faixa etária entre 18 e 35 anos, apresentar resposta negativa em todas as perguntas do questionário "ParQ", descrito por Pollock e Wilmore ${ }^{14}$, e classificação máxima de risco coronariano até nível médio no questionário proposto por "Michigan Heart Association"15. Também não deveriam ter comprometimento renal ou qualquer comorbidade e não ser consumidores de fármaco de ação diurética.

Foram adotados como critérios adicionais de seleção para o grupo de indivíduos ativos não atletas, a realização de exercício de caráter não competitivo por, no mínimo, três vezes por semana, com tempo de treino diário usualmente de 60 minutos e apresentar $\mathrm{VO}_{2 \text { máx }}$ maior ou igual 42,5 mL (kg.min)-1 e inferior a $65 \mathrm{~mL}$ (kg.min) ${ }^{-1}$. Para os avaliados do segundo grupo classificados como atletas corredores de fundo, deveriam estar submetidos a um período regular de treinamento pelo menos nos últimos dois anos, com frequência semanal variando entre 4 e 7 dias, variando em função da periodização do treino, e tempo diário usualmente superior a 60 minutos, tendo um $V_{2}$ max mínimo de $61 \mathrm{ml}$ (kg.min)-1 . Destaca-se que não ocorreu exclusão de nenhum indivíduo em nenhum dos dois grupos de comparação. As características do grupo são apresentadas na Tabela 1.

\section{Procedimentos experimentais}

Os avaliados foram submetidos a um registro antropométrico ${ }^{16}$ composto pela aferição do peso corporal, estatura e estimativa do percentual de gordura por meio da espessura das dobras cutâneas. A capacidade cardiorrespiratória foi estimada pelo teste de Cooper ${ }^{17}$, considerando como critérios para considerar o teste como máximo ter atingido nas últimas duas voltas a frequência cardíaca máxima prevista pela equação de Tanaka et al. ${ }^{18}$ que foi validada por Marins \& Fernandez ${ }^{19}$ para o uso em teste de campo para a população com o mesmo grupo etário. Também foi empregado o índice de percepção de esforço, conhecido como escala de Borg obtendo-se um valor entre 18 e 20.

Para avaliar a perda mineral no suor, os indivíduos foram submetidos a 80 minutos de exercício submáximo em esteira rolante com velocidade máxima de até $18 \mathrm{~km} / \mathrm{h}$ e inclinação de 6\%. Foi estabelecido como elemento de controle da intensidade uma faixa de trabalho entre 75-85\% da frequência cardíaca máxima $\left(F C_{\text {Máx }}\right)$, obtida no teste máximo de Cooper ${ }^{17}$, e a frequência cardíaca de repouso ( $\left.\mathrm{FC}_{\text {rep }}\right)$, permitindo, assim, o cálculo da zona alvo, considerando a frequência cardíaca de reserva, pela equação proposta por Karvonen et al. ${ }^{20}$ que é $F C T=\%\left(F C_{\text {Máx }}-F C_{\text {rep }}\right)+F C_{\text {rep. }}$. Essa estratégia buscava igualar as cargas internas de exercício físico proposto tomando como referência a resposta cardíaca do avaliado.

Durante toda a sessão de teste, a frequência cardíaca foi monitorada com o uso de monitor Polar ${ }^{\circledast}$ Acurex Plus ${ }^{\circledast}$ (Finlândia), e caso, ultrapassa-se a faixa estabelecida, a velocidade da esteira era regulada, para mais ou para menos, com a finalidade de adequar a faixa de trabalho proposta entre $75-85 \%$ de zona alvo.

Os testes foram realizados durante o dia, tendo como principal referência para iniciar o protocolo um valor da gravidade específica da urina (GEU) menor que 1,02021, verificada por refratômetro portátil (Uridens Inlab ${ }^{\circledast}$, Brasil).

Os voluntários foram orientados a se absterem do uso de álcool e de qualquer atividade física vigorosa por um período de 24 horas, anteriormente à situação experimental, assim como ingerir 400 ml de água duas horas antes do teste.

Durante o teste, estabeleceu-se uma hidratação programada a cada 15 minutos, com consumo de $3 \mathrm{ml}$ de água/Kg de peso corporal, mantendo uma condição de hidratação constante, semelhante ao estudo de Saat et al. ${ }^{22}$.

Foi coletado em três regiões corporais $2 \mathrm{~mL}$ de suor, em intervalos regulares de 20 minutos ao longo dos 80 minutos de exercício, dando assim uma característica de intermitência à corrida. As regiões selecionadas foram: a) peito, correspondendo ao espaço anatômico entre os dois mamilos; b) costas, região torácica, guiado pelo espaço anatômico entre as escápulas e c) costas, região lombar, referenciada pela $1^{\text {a a }} 5^{\text {a }}$ vértebra lombar. O modelo baseou-se na indicação de diferença nas concentrações dos minerais entre as regiões corporais 11,22 $^{11}$

Tabela 1. Características físicas e fisiológicas do grupo de avaliados.

\begin{tabular}{|c|c|c|c|c|}
\hline \multirow{2}{*}{ Parâmetros } & \multicolumn{2}{|c|}{ Atletas (n: 15) } & \multicolumn{2}{|c|}{ Ativos (n: 15) } \\
\hline & Média \pm DP & Min - Max & Média $\pm \mathrm{DP}$ & Min - Max \\
\hline Idade (anos) & $25,30 \pm 2,40$ & $22,00-29,00$ & $23,10 \pm 4,30$ & $19,00-32,00$ \\
\hline Peso (kg) & $59,60^{*} \pm 6,48$ & $48,00-73,20$ & $74,30^{*} \pm 12,6$ & $51,10-95,30$ \\
\hline Estatura (cm) & $170,90^{*} \pm 8,10$ & $158,00-188,00$ & $175,70^{*} \pm 9,50$ & $158,50-192,00$ \\
\hline$\% \mathrm{G}$ & $5,40^{*} \pm 1,90$ & $3,50-8,30$ & $10,10^{*} \pm 6,80$ & $3,00-22,80$ \\
\hline $\mathrm{SC}\left(\mathrm{m}^{2}\right)$ & $1,69 \pm 0,13$ & $1,45-1,94$ & $1,88 \pm 0,19$ & $1,50-2,18$ \\
\hline $\begin{array}{c}\text { PA sistólica } \\
\text { (mmHg) }\end{array}$ & $110,00 \pm 10,70$ & $90,00-130,00$ & $111,30 \pm 10,10$ & $100,00-135,00$ \\
\hline $\begin{array}{c}\text { PA diastólica } \\
(\mathrm{mmHg})\end{array}$ & $71,00 \pm 10,40$ & $50,00-80,00$ & $73,00 \pm 6,50$ & $60,00-80,00$ \\
\hline $\mathrm{FCr}(\mathrm{bpm})$ & $51,90 \pm 8,20$ & $38,00-65,00$ & $54,50 \pm 9,50$ & $41,00-78,00$ \\
\hline $\begin{array}{c}\mathrm{VO}_{2 \operatorname{máx}} \\
(\mathrm{mL} / \mathrm{kg} \mathrm{min})^{-1}\end{array}$ & $68,00^{*} \pm 5,40$ & $61,00-75,50$ & $50,30^{*} \pm 6,30$ & $42,50-64,30$ \\
\hline
\end{tabular}

*Diferença significativa ( $p<0.05)$ entre atletas e ativos; DP: desvio padrão da média; Min: valor mínimo; Max: valo máximo; \% G: percentual de gordura; SC: superfície corporal; PA: pressão arterial; FCr: frequência cardíaca de repouso; 
Adotou-se a técnica de coleta direta do suor, seguindo as orientações propostas por Marins ${ }^{23}$ e Falk et al. ${ }^{24}$ com modificações de acordo com a especificidade do exercício. Limpou-se a pele com água deionizada antes do inicio do exercício e a cada 10 minutos anteriores à coleta da amostra de suor. A amostra obtida foi armazenada em recipiente Eppendorf previamente desmineralizado.

Os minerais foram avaliados por espectrometria de absorção atômica, em espectrofotômetro modelo Spectr AA 220 Fs, Varian ${ }^{\oplus}$ (Darmstadt, Hessen, Alemanha). Foram realizadas diluições apropriadas à faixa de leitura do equipamento.

\section{Análise estatística}

Os dados foram submetidos ao teste de normalidade de KolmogorovSmirnov, a fim de utilizar estatística paramétrica ou não paramétrica, sendo apresentados para melhor caracterizar o grupo, em medidas de tendência central e de dispersão.

Para análise da idade, estatura, peso corporal (PC), frequência cardíaca de repouso $(\mathrm{FCr})$, pressão arterial $(\mathrm{PA})$, capacidade respiratória $\left(\mathrm{VO}_{2 \text { máx }}\right)$ e consumo de líquido utilizou-se o teste $t$ de Student. Para o percentual de gordura (\%G), superfície corporal (SC), perda mineral no suor entre os grupos, utilizou-se o teste de Mann - Whitney.

O teste de Kruskal Wallis complementado por Dunn's foi utilizado para avaliação da diferença na excreção dos minerais nas diferentes regiões de coleta, enquanto o teste de Friedman foi utilizado para comparar o efeito tempo.

Utilizou-se para análise dos dados o software Sigma Stat 3.0 (California, EUA), considerando como estatisticamente significante $p<0,05$.

\section{RESULTADOS}

A Tabela 1 apresenta as características dos avaliados. Não houve diferença estatística significativa entre eles para os parâmetros PA, FCr, SC e idade, o que demonstra a homogeneidade da amostra. Porém, verificou-se diferença significativa no $\mathrm{PC}$, altura, $\% \mathrm{G}$ e VO $\mathrm{V}_{2 \text { máx }}$ demonstrando diferença entre o nível de condicionamento dos grupos, o que é essencial para comparação.

As condições térmicas em que ocorreram os testes foram temperatura ambiente para os atletas de $21,9 \pm 1,5^{\circ} \mathrm{C}$ com umidade relativa do ar de $89,2 \pm 5,6 \%$. Para os indivíduos ativos, a situação foi temperatura de $21,8 \pm 1,6^{\circ} \mathrm{C}$ com umidade de $93,2 \pm 3,5 \%$, não havendo diferença significativa entre as situações.

As Tabelas 2, 3 e 4 apresentam, respectivamente, a concentração de $\mathrm{Ca}^{++}, \mathrm{Mg}^{++}$e Fe ${ }^{++}$, encontrada nas três regiões corporais analisadas, de acordo com o nível de condicionamento dos indivíduos.

O nível de condicionamento não promoveu diferença estatística significativa em nenhuma região corporal para os minerais investigados.

Para o $\mathrm{Ca}^{++}$também não se observou diferença estatística significativa na sua excreção ao longo do tempo para nenhum dos grupos. No entanto, quando foi avaliada sua excreção em função da região corporal, observou-se entre os atletas diferença significativa na parcial 60 minutos entre as regiões peito vs. costas região torácica. Para os ativos, diferença estatística significativa ocorreu na parcial 40 minutos entre peito vs. costas região torácica e costas região torácica vs. costas região lombar. Tanto para os 60 minutos quanto para os 80 minutos, observou-se diferença estatística entre costas região torácica vs. costas região lombar.

Com o $\mathrm{Mg}^{++}$o efeito tempo foi fator decisivo para modificações em ambos os grupos de avaliados, em todas as regiões corporais (Tabela 3), com tendência à diluição em ambos os grupos, com exceção das costas região lombar entre os ativos, em que a tendência foi de manutenção da concentração.

A região corporal que apresentou maior concentração do $\mathrm{Mg}^{++}$entre os atletas foi o peito, mas com diferença significativa apenas nas parciais 40 e 60 minutos Já, entre os ativos, ocorreu grande variação na concentração dos minerais perdidos nas diferentes regiões, dependendo do momento analisado, não sendo possível estabelecer a região de maior excreção.

O $\mathrm{Fe}^{++}$apresentou tendência de diluir-se em ambos os grupos ao longo do tempo de exercício, embora a diferença não tenha sido observada em todas as regiões e em todos os momentos de atividade.

Quando foram comparadas as diferentes regiões analisadas, houve diferença significativa entre os atletas apenas na parcial 80 minutos entre o peito vs. costas região lombar. Já, para os ativos, a diferença ocorrida foi na parcial 60 minutos entre peito vs. costas região torácica.

O consumo de líquido estipulado durante o teste foi de $3 \mathrm{~mL}$ de água/ $\mathrm{kg}$ de PC a cada 15 minutos, o que totalizou 0,89 $\pm 0,11 \mathrm{ml} \mathrm{e} \mathrm{1,124 \pm 0,173} \mathrm{ml}$ para os atletas e ativos, respectivamente, com diferença estatística entre estes resultados ( $p=0,001)$. Esta diferença se deve ao fato do consumo de líquido ter sido proporcional ao peso corporal do avaliado e como os indivíduos ativos eram mais pesados que os atletas consumiam mais líquidos. Quando se considera a perda hídrica relativa em percentual após cada experimento foram observados uma redução de 2,15 \pm 0,73\% para o grupo de atletas e de 1,04 \pm 0,67\% para o grupo de sujeitos ativos. Considera-se como valor crítico de desidratação durante um exercício quando a perda hídrica é maior do que $2 \%{ }^{21}$ o que aponta que a hidratação oferecida durante o período de exercício foi suficiente para manter um estado de homeostase hídrica em ambos os grupos em níveis ótimos.

\section{DISCUSSÃO}

O principal achado deste estudo foi que o nível de condicionamento (atletas corredores de fundo vs. indivíduos ativos não atletas) não promoveu diferença significativa na perda dos minerais em nenhuma das regiões corporais. Isto indica que o condicionamento não foi capaz de modular a perda destes elementos no suor. É possível teorizar que os sujeitos ativos, mas sem um nível de carga de treinamento como atletas, também possam gerar estímulos adaptativos nos ajustes termorregulativos ao ponto de gerar mecanismos de filtragem de eletrólitos nas glândulas sudoríparas de forma similar ao de atletas. Existem evidências que sujeitos treinados possuem respostas termorregulativas diferentes no tempo de produção

Tabela 2. Concentração de cálcio (mmol..$\left.^{-1}\right)$ no suor nas três regiões corporais ao longo de 80 minutos de exercício entre os atletas e ativos.

\begin{tabular}{|c|c|c|c|c|c|c|c|c|c|c|c|c|}
\hline \multirow[b]{3}{*}{$\mathrm{T}$} & \multicolumn{6}{|c|}{ Atletas } & \multicolumn{6}{|c|}{ Ativos } \\
\hline & \multicolumn{2}{|c|}{ Peito } & \multicolumn{2}{|c|}{ Costa torácica } & \multicolumn{2}{|c|}{ Costa lombar } & \multicolumn{2}{|c|}{ Peito } & \multicolumn{2}{|c|}{ Costa torácica } & \multicolumn{2}{|c|}{ Costa lombar } \\
\hline & $\begin{array}{c}\text { Mediana } \\
(X \pm D P)\end{array}$ & Min - Máx & $\begin{array}{c}\text { Mediana } \\
(X \pm D P)\end{array}$ & Min - Máx & $\begin{array}{c}\text { Mediana } \\
(X \pm D P)\end{array}$ & Min - Máx & $\begin{array}{c}\text { Mediana } \\
(X \pm D P)\end{array}$ & Min - Máx & $\begin{array}{c}\text { Mediana } \\
(X \pm D P)\end{array}$ & Min - Máx & $\begin{array}{c}\text { Mediana } \\
(X \pm D P)\end{array}$ & Min - Máx \\
\hline 20 & $\begin{array}{c}0,33 \\
(0,55 \pm 0,50)\end{array}$ & $\begin{array}{c}0,050- \\
1,358\end{array}$ & $\begin{array}{c}0,17 \\
(0,38 \pm 0,35)\end{array}$ & $\begin{array}{c}0,076- \\
1,206\end{array}$ & $\begin{array}{c}0,317 \\
(0,5 \pm 0,5)\end{array}$ & $\begin{array}{c}0,154- \\
1,754\end{array}$ & $\begin{array}{c}0,63 \\
(0,64 \pm 0,43) \\
\end{array}$ & $\begin{array}{c}0,088- \\
1,622\end{array}$ & $\begin{array}{c}0,40 \\
(0,49 \pm 0,41)\end{array}$ & $\begin{array}{c}0,029- \\
1,416\end{array}$ & $\begin{array}{c}0,53 \\
(0,52 \pm 0,28)\end{array}$ & $\begin{array}{c}0,096- \\
0,965\end{array}$ \\
\hline 40 & $\begin{array}{c}0,2 \\
(0,34 \pm 0,36)\end{array}$ & $\begin{array}{c}0,030- \\
1,320 \\
\end{array}$ & $\begin{array}{c}0,19 \\
(0,21 \pm 0,2) \\
\end{array}$ & $\begin{array}{c}0,025- \\
0,742 \\
\end{array}$ & $\begin{array}{c}0,262 \\
(0,29 \pm 0,29) \\
\end{array}$ & $\begin{array}{c}0,074- \\
1,255 \\
\end{array}$ & $\begin{array}{c}0,23^{\mathrm{A}} \\
(0,36 \pm 0,3) \\
\end{array}$ & $\begin{array}{c}0,093- \\
1,270 \\
\end{array}$ & $\begin{array}{c}0,12^{\mathrm{B}} \\
(0,21 \pm 0,18)\end{array}$ & $\begin{array}{c}0,070- \\
0,662\end{array}$ & $\begin{array}{c}0,28 \\
(0,31 \pm 0,17)\end{array}$ & $\begin{array}{c}0,114- \\
0,727\end{array}$ \\
\hline 60 & $\begin{array}{c}0,22^{*} \\
(0,31 \pm 0,26) \\
\end{array}$ & $0,00-0,763$ & $\begin{array}{c}0,16^{*} \\
(0,15 \pm 0,08) \\
\end{array}$ & $\begin{array}{c}0,021- \\
0,288 \\
\end{array}$ & $\begin{array}{c}0,12 \\
(0,23 \pm 0,11) \\
\end{array}$ & $0,088-0,460$ & $\begin{array}{c}0,29 \\
(0,31 \pm 0,24) \\
\end{array}$ & $\begin{array}{c}0,043- \\
1,048 \\
\end{array}$ & $\begin{array}{c}0,15^{\mathrm{B}} \\
(0,22 \pm 0,2)\end{array}$ & $\begin{array}{c}0,048- \\
0,752 \\
\end{array}$ & $\begin{array}{c}0,29 \\
(0,34 \pm 0,21) \\
\end{array}$ & $\begin{array}{c}0,054- \\
0,818 \\
\end{array}$ \\
\hline 80 & $\begin{array}{c}0,19 \\
(0,26 \pm 0,19)\end{array}$ & $\begin{array}{c}0,027- \\
0,631 \\
\end{array}$ & $\begin{array}{c}0,15 \\
(0,17 \pm 0,08)\end{array}$ & $\begin{array}{c}0,048- \\
0,316 \\
\end{array}$ & $\begin{array}{c}0,205 \\
(0,23 \pm 0,1) \\
\end{array}$ & $\begin{array}{c}0,086- \\
0,399\end{array}$ & $\begin{array}{c}0,29 \\
(0,29 \pm 0,17)\end{array}$ & $\begin{array}{c}0,078- \\
0,788\end{array}$ & $\begin{array}{c}0,18^{\mathrm{B}} \\
(0,25 \pm 0,21)\end{array}$ & $\begin{array}{c}0,052- \\
0,864\end{array}$ & $\begin{array}{c}0,30 \\
(0,35 \pm 0,2)\end{array}$ & $\begin{array}{c}0,085- \\
0,897\end{array}$ \\
\hline
\end{tabular}


Tabela 3. Concentração de magnésio (mmol..-1) no suor nas três regiões corporais ao longo de 80 minutos de exercício entre os atletas e ativos.

\begin{tabular}{|c|c|c|c|c|c|c|c|c|c|c|c|c|}
\hline \multirow{3}{*}{$\mathrm{T}$} & \multicolumn{6}{|c|}{ Atletas } & \multicolumn{6}{|c|}{ Ativos } \\
\hline & \multicolumn{2}{|c|}{ Peito } & \multicolumn{2}{|c|}{ Costa torácica } & \multicolumn{2}{|c|}{ Costa lombar } & \multicolumn{2}{|c|}{ Peito } & \multicolumn{2}{|c|}{ Costa torácica } & \multicolumn{2}{|c|}{ Costa lombar } \\
\hline & $\begin{array}{c}\text { Mediana } \\
(\mathrm{X} \pm \mathrm{DP})\end{array}$ & Min - Máx & $\begin{array}{c}\text { Mediana } \\
(X \pm D P)\end{array}$ & Min-Máx & $\begin{array}{r}\text { Mediana } \\
(X \pm D P)\end{array}$ & Min - Máx & $\begin{array}{c}\text { Mediana } \\
(X \pm D P)\end{array}$ & Min-Máx & $\begin{array}{r}\text { Mediana } \\
(X \pm D P)\end{array}$ & Min - Máx & $\begin{array}{r}\text { Mediana } \\
(X \pm D P)\end{array}$ & Min - Máx \\
\hline 20 & $\begin{array}{c}0,23^{\mathrm{AB}} \\
(0,19 \pm 0,1)\end{array}$ & $\begin{array}{c}0,051- \\
0,337 \\
\end{array}$ & $\begin{array}{c}0,10^{\mathrm{CD}} \\
(0,13 \pm 0,12) \\
\end{array}$ & $\begin{array}{c}0,032- \\
0,498 \\
\end{array}$ & $\begin{array}{c}0,11^{\mathrm{EF}} \\
(0,13 \pm 0,08) \\
\end{array}$ & $\begin{array}{c}0,040- \\
0,311 \\
\end{array}$ & $\begin{array}{c}0,211^{\text {GHI\& }} \\
(0,24 \pm 0,17)\end{array}$ & $\begin{array}{c}0,038- \\
0,731 \\
\end{array}$ & $\begin{array}{c}0,220^{\text {KLM }} \\
(0,2 \pm 0,15)\end{array}$ & $\begin{array}{c}0,025- \\
0,497 \\
\end{array}$ & $\begin{array}{c}0,13^{\mathrm{NO}} \\
(0,17 \pm 0,13)\end{array}$ & $\begin{array}{c}0,016- \\
0,395 \\
\end{array}$ \\
\hline 40 & $\begin{array}{c}0,09^{*} \\
(0,12 \pm 0,11) \\
\end{array}$ & $\begin{array}{c}0,033- \\
0,461 \\
\end{array}$ & $\begin{array}{c}0,04 \\
(0,08 \pm 0,1) \\
\end{array}$ & $\begin{array}{c}0,001- \\
0,417 \\
\end{array}$ & $\begin{array}{c}0,08 \\
(0,09 \pm 0,08) \\
\end{array}$ & $\begin{array}{c}0,021- \\
0,314 \\
\end{array}$ & $\begin{array}{c}0,115^{\jmath} \\
(0,12 \pm 0,08) \\
\end{array}$ & $\begin{array}{c}0,020- \\
0,289 \\
\end{array}$ & $\begin{array}{c}0,059 \\
(0,07 \pm 0,07) \\
\end{array}$ & $\begin{array}{c}0,002- \\
0,248 \\
\end{array}$ & $\begin{array}{c}0,09 \\
(0,1 \pm 0,07) \\
\end{array}$ & $\begin{array}{c}0,008- \\
0,270 \\
\end{array}$ \\
\hline 60 & $\begin{array}{c}0,08^{* \#} \\
(0,11 \pm 0,08) \\
\end{array}$ & $\begin{array}{c}0,024- \\
0,298 \\
\end{array}$ & $\begin{array}{c}0,04 \\
(0,06 \pm 0,07) \\
\end{array}$ & $\begin{array}{c}0,000- \\
0,270 \\
\end{array}$ & $\begin{array}{c}0,06 \\
(0,07 \pm 0,04) \\
\end{array}$ & $\begin{array}{c}0,005- \\
0,132 \\
\end{array}$ & $\begin{array}{c}0,075 \\
(0,11 \pm 0,11 \\
\end{array}$ & $\begin{array}{c}0,024- \\
0,489 \\
\end{array}$ & $\begin{array}{c}0,048^{5} \\
(0,09 \pm 0,13) \\
\end{array}$ & $\begin{array}{c}0,007- \\
0,528 \\
\end{array}$ & $\begin{array}{c}0,12 \\
(0,11 \pm 0,08) \\
\end{array}$ & $\begin{array}{c}0,003- \\
0,278 \\
\end{array}$ \\
\hline 80 & $\begin{array}{c}0,07 \\
(0,08 \pm 0,06)\end{array}$ & $\begin{array}{c}0,023- \\
0,238\end{array}$ & $\begin{array}{c}0,03 \\
(0,05 \pm 0,05)\end{array}$ & $\begin{array}{c}0,005- \\
0,198\end{array}$ & $\begin{array}{c}0,05 \\
(0,06 \pm 0,05)\end{array}$ & $\begin{array}{c}0,004- \\
0,158\end{array}$ & $\begin{array}{c}0,061 \\
(0,08 \pm 0,05)\end{array}$ & $\begin{array}{c}0,016- \\
0,213\end{array}$ & $\begin{array}{c}0,034^{\S} \\
(0,07 \pm 0,1)\end{array}$ & $\begin{array}{c}0,005- \\
0,399\end{array}$ & $\begin{array}{c}0,10 \\
(0,1 \pm 0,08)\end{array}$ & $\begin{array}{c}0,002- \\
0,273\end{array}$ \\
\hline
\end{tabular}

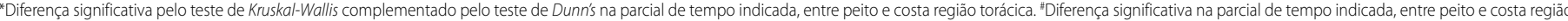

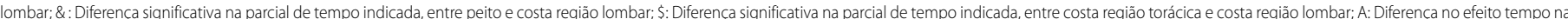

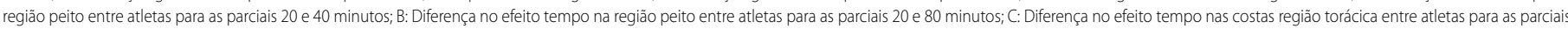

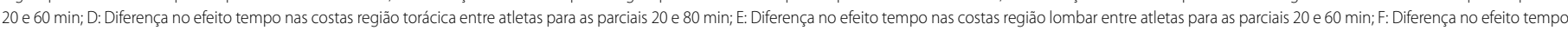

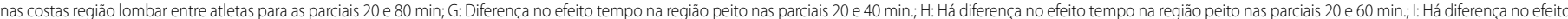

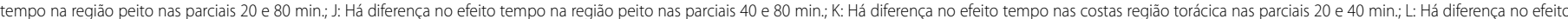

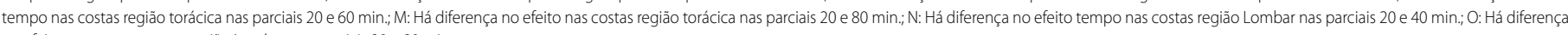
no efeito tempo nas costas regiäo Lombar nas parciais 20 e 80 min

Tabela 4. Concentração de ferro (mmol..-1) no suor nas três regiões corporais ao longo de 80 minutos de exercício entre os atletas e ativos.

\begin{tabular}{|c|c|c|c|c|c|c|c|c|c|}
\hline \multirow{3}{*}{$\mathbf{T}$} & \multicolumn{9}{|c|}{ Atletas } \\
\hline & \multicolumn{3}{|c|}{ Peito } & \multicolumn{3}{|c|}{ Costa torácica } & \multicolumn{3}{|c|}{ Costa lombar } \\
\hline & Mediana & Média \pm DP & Min - Máx & Mediana & Média \pm DP & Min - Máx & Mediana & Média \pm DP & Min - Máx \\
\hline 20 & 0,0018 & $0,0036 \pm 0,0042$ & $0,0003-0,0167$ & $0,0023^{A}$ & $0,0042 \pm 0,0017$ & $0,0002-0,0159$ & $0,003^{\mathrm{CD}}$ & $0,0039 \pm 0,0034$ & $0,0001-0,0100$ \\
\hline 40 & 0,0019 & $0,0045 \pm 0,0048$ & $0,0002-0,0149$ & $0,0015^{B}$ & $0,0019 \pm 0,0013$ & $0,0003-0,0062$ & $0,002^{\mathrm{E}}$ & $0,0027 \pm 0,0026$ & $0,0005-0,0097$ \\
\hline 60 & 0,0013 & $0,0042 \pm 0,006$ & $0,0001-0,0203$ & 0,0008 & $0,0013 \pm 0,0015$ & $0,0001-0,0047$ & 0,001 & $0,0015 \pm 0,0014$ & $0,0001-0,0056$ \\
\hline 80 & $0,0015^{\#}$ & $0,0044 \pm 0,0071$ & $0,0003-0,0276$ & 0,0005 & $0,001 \pm 0,0015$ & $0,0000-0,0061$ & 0,0006 & $0,0011 \pm 0,0011$ & $0,0001-0,0037$ \\
\hline \multirow{3}{*}{$T$} & \multicolumn{9}{|c|}{ Ativos } \\
\hline & \multicolumn{3}{|c|}{ Peito } & \multicolumn{3}{|c|}{ Costa torácica } & \multicolumn{3}{|c|}{ Costa lombar } \\
\hline & Mediana & Média \pm DP & Min - Máx & Mediana & Média \pm DP & Min - Máx & Mediana & Média \pm DP & Min - Máx \\
\hline 20 & $0,0068^{\mathrm{F}}$ & $0,0098 \pm 0,0121$ & $0,0005-0,0474$ & $0,0027^{\mathrm{H}}$ & $0,0049 \pm 0,0052$ & $0,0003-0,0172$ & $0,0040^{\prime}$ & $0,0056 \pm 0,0045$ & $0,0005-0,0152$ \\
\hline 40 & $0,0039^{G}$ & $0,0069 \pm 0,0075$ & $0,0004-0,0213$ & 0,0017 & $0,0039 \pm 0,0051$ & $0,0004-0,0161$ & 0,0020 & $0,0029 \pm 0,0029$ & $0,0003-0,0096$ \\
\hline 60 & $0,0022^{*}$ & $0,0052 \pm 0,0059$ & $0,0003-0,0198$ & 0,0013 & $0,0023 \pm 0,0034$ & $0,0000-0,0133$ & 0,0018 & $0,0038 \pm 0,006$ & $0,0001-0,0230$ \\
\hline 80 & 0,0014 & $0,0043 \pm 0,0068$ & $0,0002-0,0263$ & 0,0007 & $0,0015 \pm 0,0017$ & $0,0000-0,0043$ & 0,0018 & $0,0035 \pm 0,0039$ & $0,0000-0,0104$ \\
\hline
\end{tabular}

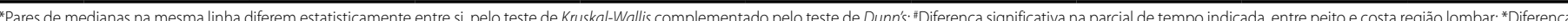

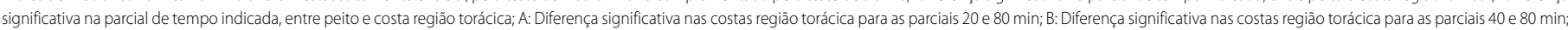

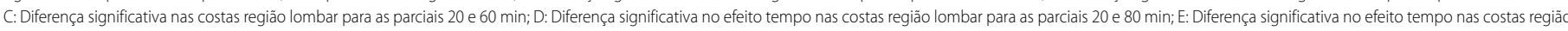

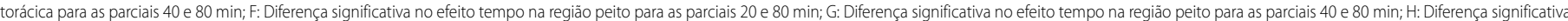
no efeito tempo nas costas regiäo torácica para as parciais 20 e $80 \mathrm{~min}$; : Diferença significativa no efeito tempo nas costas região Lombar para as parciais 20 e 80 min.

inicial de sudorese bem como sua concentração mineral por conta das adaptações ao estresse de calor que o exercício impõe ${ }^{25}$. Um nível de desidratação avançado pode ser um fator determinante na alteração da concentração eletrolítica do suor ${ }^{10}$. No presente estudo a hidratação foi proporcional em cada sujeito considerando $3 \mathrm{ml} / \mathrm{kg}$ de peso corporal. Isto impactou positivamente na manutenção da homeostase hídrica, tendo em vista que, a perda hídrica média observada foi aproximadamente de 2\%, o que garante que o procedimento adotado de hidratação foi adequado, sem expor os corredores a nível agudos de desidratação ${ }^{21}$. Assim que se considera que este fator não tenha influenciado entre os grupos.

Atletas por apresentar um melhor condicionamento acabam treinando maior número de dias na semana, ou mesmo de sessões no mesmo dia. Também se exercitam por um maior tempo, e em certas ocasiões em condições térmicas desfavoráveis de estresse térmico, aumentando a perda hídrica e mineral, necessitando repor adequadamente estes elementos.

Neste estudo, observou-se que a concentração de $\mathrm{Ca}^{++}$no suor esteve dentro da faixa de normalidade sugerida por Shirreffs e Maughan ${ }^{11}$ que varia de 0 a 2,9 mmol/L, não ocorrendo registro de valores acima destes em nenhum dos grupos.

Indivíduos ativos apresentaram excreção de $\mathrm{Ca}^{++}$no suor maior que os atletas. No entanto, esta diferença não foi estatisticamente significativa, demonstrando que o nível de condicionamento não influenciou na taxa de excreção do mineral no suor.

Böhmer ${ }^{26}$ também observou uma menor perda de $\mathrm{Ca}^{++}$entre jogadores de handebol bem treinados em comparação com jogadores com treinamento médio. Porém, o autor não revelou se esta diferença era ou não significativa.

Quanto ao efeito tempo, pôde-se observar que, diferentemente de outros estudos em que ocorreu redução significativa da concentração de $\mathrm{Ca}^{++}$ao longo do tempo de exercício ${ }^{23,26}$, neste não se verificou diminuição da mesma ao longo da atividade. Isto pode ser um fator contribuidor para redução das reservas corporais entre indivíduos que se exercitam por longo tempo e não realizam reposição adequada, podendo, pelo efeito cumulativo, acarretar comprometimento ósseo.

Esta alteração no comportamento do $\mathrm{Ca}^{++}$em diferentes estudos pode estar relacionada à diferença populacional, pois em ambos os estudos, os avaliados eram de origem europeia, deixando assim em aberto a questão da aclimatação ou etnia.

Entre as regiões corporais avaliadas, observou-se diferença significativa na excreção do $\mathrm{Ca}^{++}$de acordo com o momento e local avaliado. As diferenças foram mais facilmente encontradas entre os ativos (Tabela 2). Riano et al. ${ }^{27}$ quando investigaram a concentração de $\mathrm{Ca}^{++}$no suor nas 
regiões costas, braços e peito também verificaram diferença significativa de acordo com a região analisada.

Quanto ao $\mathrm{Mg}^{++}$, verificou-se que o nível de condicionamento não ocasionou diferença significativa na sua perda no suor, inferindo-se que o melhor condicionamento físico não minimiza as perdas como ocorre com outros minerais ${ }^{26}$. 0 Mg $^{++}$é um mineral intracelular e sua carência é extremamente incomum. Somente em casos bem avançados de desnutrição, condutas nutricionais muito radicais, ou certas patologias são possíveis observar carência deste mineral. O presente estudo apontou que a perda deste mineral de forma aguda é semelhante (atletas corredores de fundo vs. indivíduos ativos não atletas). Contudo existem atletas que participam de provas de ultraendurance que geram perdas hídricas minerais elevadas e de forma crônica gerando uma perda contínua do $\mathrm{Mg}^{++}$, devendo ser monitorado, a fim de se avaliar qual a magnitude de seus estoques corporais e qual melhor estratégia para se estabelecer o equilíbrio deste nutriente.

Beller et al. ${ }^{28}$ verificaram que oito homens não aclimatados, exercitando-se por 90 minutos, no calor, tiveram redução significativa dos níveis de $\mathrm{Mg}^{++}$no plasma. Porém, eles afirmaram que esta redução não poderia ser totalmente explicada pela excreção do mineral no suor, uma vez que esta foi, em média, de 0,28mEq/L, quantidade considerada dentro da faixa de normalidade. Analisando o estudo, realmente a excreção do $\mathrm{Mg}^{++}$no suor pode, não ter sido o principal fator que acarretou diminuição plasmática deste, mas, certamente, foi um forte contribuidor.

Quanto ao efeito tempo, em ambos os grupos, ocorreu tendência à diminuição da concentração de $\mathrm{Mg}^{++}$no suor no decorrer do exercício, exceção apenas para costas na região lombar entre os indivíduos ativos. Este achado demonstra que a realização de uma única medida e sua extrapolação para o percentual de perda do mineral no suor para todo o tempo de exercício pode levar a uma superestimação dos valores perdidos, conduzindo a estratégias de reposição inadequadas.

Böhmer ${ }^{26}$ avaliando os jogadores de handebol, também verificou redução na concentração média de $\mathrm{Mg}^{++}$ao longo dos 75 minutos de exercício, tanto em mulheres quanto em homens. Entretanto, não revelou se a diferença ocorrida era significativa.

Conforme observado em outros estudos, com diferentes minerais 29,30 houve diferença significativa entre as regiões corporais em que o suor foi coletado, sendo que entre os atletas, o peito foi a região de maior perda, aos 40 e 60 minutos. Entre os ativos não foi possível estabelecer a região com maior excreção, pois dependendo do momento analisado, a região com maior perda do mineral alterava-se.

Quando se compara a perda de $\mathrm{Mg}^{++}$ocorrida neste estudo em ambos os grupos com a perda apresentada por outros autores ${ }^{26,31}$ verifica-se que estas foram semelhantes, assim como estiveram dentro da faixa de normalidade descrita por Shirreffs e Maughan ${ }^{11}$, que corresponde a 0-1,5 mmol.

No que se refere ao $\mathrm{Fe}^{++}$, há relatos consistentes na literatura de baixa ingestão deste mineral entre atletas ${ }^{6}$, sendo um mineral que apresenta alta frequência de deficiência ${ }^{32}$. Embora o suor não seja uma das suas principais vias de excreção, avaliar sua perda no suor em situação de exercício é importante, uma vez que uma perda excessiva pode contribuir para a deficiência encontrada em grupos de atletas ${ }^{33}$.

Neste estudo, não houve diferença significativa na excreção de $\mathrm{Fe}^{++}$ entre os grupos (Tabela 4), sendo que um importante achado deste foi a existência de diferença significativa entre as regiões analisadas apenas para os atletas aos 80 minutos entre peito e costas região lombar e para os ativos na parcial de 60 minutos entre peito e costas região torácica. A não existência de diferença significativa entre as demais regiões, em cada parcial avaliada, demonstra que, para o $\mathrm{Fe}^{++}$, obedecendo aos princípios adotados neste estudo, a região corporal analisada não foi fator determinante para as diferenças na concentração do mineral no suor. Isto permite supor que a análise de uma única região pode ser realizada para estabelecimento da concentração do mineral no exercício.

No estudo de DeRuisseau et al. ${ }^{34}$, também não houve diferença significativa na concentração de $\mathrm{Fe}^{++}$no suor entre os braços direito e esquerdo, revelando, mais uma vez que, os diferentes minerais apresentam comportamentos distintos.

Alguns estudos têm demonstrado que a concentração do $\mathrm{Fe}^{++}$no suor é dependente da taxa de sudorese ${ }^{33,35}$, sendo que esta relação é inversa, ou seja, quanto maior a taxa de sudorese menor seria a concentração do $\mathrm{Fe}^{++}$no suor. Desta forma, verificar a taxa de sudorese é importante para o planejamento dietético.

Embora a perda de $\mathrm{Fe}^{++}$no suor seja pequena em relação a outros elementos, e ainda sofra diluição no decorrer do tempo de exercício, em atividades com duração prolongada como Ironman, ou provas de aventura que duram vários dias, as perdas podem ser consideráveis, podendo levar a um balanço negativo de ferro.

A perda de $\mathrm{Fe}^{++}$no suor atuando isoladamente dificilmente poderá gerar deficiência deste nutriente no organismo. Porém, quando altas perdas ocorridas em dias sucessivos estão associadas à ingestão inadequada, dietas com baixa biodisponibilidade, perda excessiva decorrente da menstruação, perdas gastrintestinais, dietas vegetarianas e excreção aumentada de Fe ${ }^{++}$ na urina, há condições propícias para o desenvolvimento de deficiências.

Considerando que a perda de Fe $\mathrm{F}^{++}$no suor em ambos os grupos foi variável (Tabela 4), é necessária uma análise individual para que suas perdas no suor não sejam mais um contribuidor para um balanço negativo deste nutriente no organismo. Para indivíduos que possuem um grande volume de treinos diários, a suplementação de $\mathrm{Fe}^{++}$pode ser recomendada a fim de manter as reservas corporais equilibradas.

Uma possível limitação do estudo foi não ter padronizado a densidade de pelos na região de coleta de suor, o que pode produzir alguma variação no resultado, abrindo mais uma possibilidade de avaliações futuras se a densidade de pelos interfere ou não na concentração mineral do suor.

\section{CONCLUSÕES}

O principal achado deste estudo foi que o nível de condicionamento não promoveu diferença significativa nas perdas dos minerais $\mathrm{Ca}^{++}, \mathrm{Mg}^{++}$ e Fe ${ }^{++}$em nenhuma das três regiões corporais.

Entre as regiões corporais avaliadas, pode-se observar que houve diferença significativa na excreção do $\mathrm{Ca}^{++}$, enquanto que, para o $\mathrm{Mg}^{++}$, as diferenças entre as regiões variaram de acordo com o grupo avaliado. No caso do Fe, houve diferença significativa apenas em uma parcial de tempo para cada um dos grupos. Não se observou diferença significativa para o $\mathrm{Ca}^{++}$nos grupos quanto ao tempo para qualquer das regiões corporais, enquanto para o $\mathrm{Mg}^{++}$e o $\mathrm{Fe}^{++}$houve tendência à diluição à medida que o exercício progredia, diminuindo o risco da carência mineral ao longo do exercício.

Nas condições em que o exercício foi realizado, a quantidade de minerais perdidos no suor não foi suficiente para produzir perdas elevadas.

\section{AGRADECIMENTOS}

Os autores agradecem a todos os voluntários que contribuíram com o estudo, à Coordenação de Aperfeiçoamento de Pessoal de Nível Superior (CAPES) e a Fundação de Amparo à Pesquisa do Estado de Minas Gerais (FAPEMIG) pelo apoio financeiro no desenvolvimento da pesquisa.

$\overline{\text { Todos os autores declararam não haver qualquer potencial conflito }}$ de interesses referente a este artigo. 
CONTRIBUIÇÕES DOS AUTORES: Cada autor contribuiu individual e significativamente para o desenvolvimento do manuscrito. FGF (0000-0001-9836-4176)* , NMBC (00000002-4967-9937)*, AMCS (0000-0002-5361-5741)* e JCBM (0000-0003-0727-3450)* foram os principais contribuintes na concepção e desenho da pesquisa. FGF e MF (00000001-7285-0029)* obtiveram os dados, realizaram pesquisa bibliográfica, as análises estatísticas e interpretação dos dados. FGF e JCBM realizaram a redação do manuscrito. FGF, MF, NMBC, AMCS e JCBM, realizaram revisão crítica e provaram a versão final do manuscrito. *ORCID (Open Researcher and Contributor ID).

\section{REFERÊNCIAS}

1. Altman PL. Blood and other body fluids. Washington: Dorothy Dittmer; 1961.

2. Saraymen R, Kiliç E, Yazar, S. Sweat copper, zinc, iron, magnesium and chromium levels in national wrestler. J Turgut Ozal Med Cent. 2004;11(1):7-10.

3. Gomes RV, Ribeiro SML, Veibig RF, Aoki MS. Consumo alimentar e Perfil antropométrico de tenistas amadores e profissionais. Rev Bras Med Esporte. 2009;15(6):436-40.

4. Cabral CAC, Rosado GP, Silva CHO, Marins JCB. Diagnóstico do estado nutricional dos atletas da Equipe Olímpica Permanente de Levantamento de Peso do Comitê Olímpico Brasileiro (COB). Rev Bras Med Esporte. 2006; 12(6):345-50

5. Paschoal VC, Amancio OM. Nutritional status of Brazilian elite swimmers. Int J Sport Nutr Exerc Metab. 2004;14(1):81-94

6. Papadopoulou SK, Papadopoulou SD, Gallos GK. Macro- and micro-nutrient intake of adolescent Greek female volleyball players. Int J Sport Nutr Exerc Metab. 2002;12(1):73-80.

7. Clarkson PM, Haymes EM. Exercise and mineral status of athletes: calcium, magnesium, phosphorus, and iron. Med Sci Sports Exerc. 1995;27(6):831-43.

8. Hamouti N, Del Coso J, Estevez E, Mora-Rodrigues R. Dehydration and sodium deficit during indoor practive in elite European male team players. Eur J Sport Sci. 2010;10:329-36

9. Baker LB, Stofan JR, Hamilton AA, Horswill CA. Comparison of regional patch collection vs. whole body washdown for measuring sweat sodium and potassium loss during exercise. J Appl Physiol. 2009;107(3):887-95.

10. Morgan RM, Patterson MJ, Nimmo MA. Acute effects of dehydration on sweat composition in men during prolonged exercise in the heat. Acta Physiol Scand. 2004;182(1):37-43.

11. Shirreffs SM, Maughan RJ. Whole body sweat collection in humans: an improved method with preliminary data on electrolyte content. J Appl Physiol. 1997;82(1):336-41.

12. Inoue Y, Nakao M, Araki T, Murakami H. Regional differences in the sweating responses of older and younger men. J Appl Physiol. 1991;71(6): 2453-9.

13. Amano T, Gerrett N, Inoue Y, Nishiyasu T, Havenith G, Kondo N. Determination of the maximum rate of eccrine sweat glands'ion reabsorption using the galvanic skin conductance to local sweat rate relationship. Eur J Appl Physiol. 2016;116(2):281-90.

14. Pollock ML. Exercício na saúde e na doença: avaliação e prescrição para prevenção e reabilitação. $2^{\text {a }}$ ed. Rio de Janeiro: Medsi; 1993.

15. McArdle WD, Katch Fl, Katch VL. fisiologia do exercício energia, nutrição e desempenho humano. $5^{\mathrm{a}}$ ed. Rio de Janeiro: Guanabara Koogan, 2003.

16. Marfell-Jones M, Olds T, Stewart A, Carter UL. International standards for anthropometric assessment: ISAK; South Africa; 2006

17. Cooper KH. O programa aeróbio para o bem-estar total. Rio de Janeiro: Nórdica; 1982.

18. Tanaka HK, Monahan KD, Seals DR. Age-Predicted maximal heart rate revisited. J Am Coll Cardiol. 2001;37(1):153-6.
19. Marins JC, Fernandez MD. Employment of equations to esteem the maximum heart hate in running for young sportsman. Arch Med Deporte. 2007;24(118):112-20.

20. Karvonen MJ, Kentala E, Mustala O. The effects of training heart rate: a longitudinal study. Ann Med Exp Biol Fenn. 1957:35(3):307-15

21. Casa DJ, Armstrong LE, Hillman SK, Montain SJ, Reiff RV, Rich BS, et al. National athletic trainers'association position statement: fluid replacement for athletes. J AthI Train. 2000;35(2):212-24.

22. Saat M, Tochihara Y, Hashiguchi N, Sirisinghe RG, Fujita M, Chou CM. Effects of exercise in the heat on thermoregulation of Japanese and Malaysian males. J Physiol Anthropol Appl Human Sci. 2005;24(4):267-75.

23. Marins JCB. Estudio comparativo de diferentes procedimientos de hidratación durante un ejercicio de larga duración (Tesis Doctoral). Murcia: Departamento de Fisiología y Farmacología. Universidad de Murcia; 2000

24. Falk B, Bar-or O, MacDougall JD, McGillis L, Calvert R, Meyer F. Sweat lactate in exercising children and adolescents of varying physical maturity. J Appl Physiol. 1991;71(5):1735-40.

25. Périard JD, Racinais S, Sawka MN. Adaptations and mechanisms of human heat acclimation: applications for competitive athletes and sports. Scand J Med Sci Sports. 2015;25(Suppl 1):20-38.

26. Böhmer D. Loss of electrolyte by sweat in sport. Ed. Wilmoth S. In: Olympic Scientific Congress. Champaign: Human Kinetics; 1986

27. Rianon N, Feeback D, Wood R, Driscoll T, Shackelford L, LeBlanc A. Monitoring sweat calcium using skin patches. Calcif Tissue Int. 2003;72(6): 694-7.

28. Beller GA, Maher JT, Hartley LH, Bass, DE, Wacker W E. Changes in serum and sweat magnesium levels during work in the heat. Aviat Space Environ Med.1975;46(5):709-12.

29. Takamata A, Yoshida T, Nishida N, Morimoto T. Relationship of osmotic inhibition in thermoregulatory responses and sweat sodium concentration in humans. Am J Physiol Regul Integr Comp Physiol. 2001;280(3):R623-9.

30. Patterson MJ, Galloway SD, Nimmo MA. Variations in regional sweat composition in normal human males. Exp Physiol. 2000;85(6):869-75.

31. Palacios C, Wigertz K., Weaver CM. Comparison of 24 hour whole body versus patch tests for estimating body surface electrolyte losses. Int J Sport Nutr Exerc Metab. 2003;13(4):479-88.

32. Dubnov G, Constantini NW. Prevalence of iron depletion and anemia in top-level basketball players. In J Sport Nutr Exerc Metab. 2004;14(1):30-7.

33. Waller MF, Haymes, EM. The effects of heat and exercise on sweat iron loss. Med Sci Sports Exerc.1996;28(2):197-203.

34. DeRuisseau KC, Cheuvront SN, Haymes EM, Sharp RG. Sweat iron and zinc losses during prolonged exercise. Int J Sport Nutr Exerc Metab. 2002 12(4):428-37.

35. Lamanca JJ, Haymes, EM, Daly JA, Moffatt RJ, Waller MF. Sweat iron loss of male and female runners during exercise. Int J Sports Med, 1988;9(1):52-5. 